\title{
Formation of the Cost Parameters of Engineering Survey for Construction of the Energy Efficient Buildings
}

\author{
Ivan Doroshin ${ }^{1, *}$ \\ ${ }^{1}$ National Research Moscow State University of Civil Engineering,26, Yaroslavskoye shosse, \\ Moscow,129337, Russia
}

\begin{abstract}
Annotation. This article considers the questions of cost parameter system formation to determine the cost of the prospecting works on the example of engineering-technical examination of intelligent buildings. Certain surveys enroll specific features for intelligent buildings. The scheme of the cost formation for the works and the necessary input data was considered. There were given recommendations on formation of prices for certain types of works in order to determine the cost of the work more precisely. The author gives the suggestions on the composition and calculation of additional coefficients that take into account the conditions of work and change their value.
\end{abstract}

\section{Introduction}

The modern condition of the construction industry is characterized by a number of global problems, such as energy and environmental issues. Their direction is determined by a complex solution of the environmental conservation problem through carrying out the researches in the field of energy saving, including the switch to the alternative inexhaustible energy sources. Up today a big number of buildings, neighborhoods and even architectural zones have been built, which were designed on the basis of various energy-saving concepts in the context of solving these problems.

In practice the "Energy efficient building" concept became the most common standard, which has energy saving as its primary. That is achieved through the application of innovative decisions, which are technically feasible, economically justified and acceptable from the environmental and social points of view.

It is worth mentioning that the directions stated above are carried out in the long-term planning time-frame, associated with environmental preservation for future generations and assessment of the payback at the expense of reduction of the energy consumption and predicted growth of the prices for energy sources. However, a tendency of expansion in the number of the complex engineering systems was observed in addition to the improvement of the technologies in the field of efficient energy-saving.

${ }^{*}$ Corresponding author: ivandoroshin@rambler.ru 


\section{Methods}

The aanalysis of the principles stated above allowed to reveal the following properties of the intelligent building system, predetermining the efficiency of the project "intelligent building" realization:

1. Minimization of the number of the system components by reducing a number of its back-up elements.

2. Optimization of the functioning mode of every intellectual building subsystem on the basis of the data interchange with other subsystems of the building.

3. Adequate and rapid response on changes in user requirements and processes occurring in the building due to its high controllability.

4. Reduction of operation timings by the high degree of protection from human error.

5. Systematization of the operation data of all engineering systems in the building thanks to the complex data collection and protection.

6. Continuous monitoring of the intelligent building subsystems condition, electronic $\log$ keeping that allows to analyze events and actions over a long period of time and plan the exploitation costs.

7. Preventive response to a failure is detection, identification and prevention of emergency situations.

8. Automated decision support in the event of occurrence of emergency situations using the expert subsystem.

The list of the stated above system properties is not exhaustive and, in the case of the detection of new ones, it must be continually updated. Fulfilment of the intellectual building benefits allows to build a support system for every stage of the real estate unit life cycle: pre-design, design, construction, operation.

To speak about the features of the formation and determination of the cost of engineering surveys and methods used for this purpose, first of all, the system of the cost parameter formation should be defined, i.e. what data and in what sequence such costs are to be determined.

Such type of works is quite diverse. There are several types of them. For instance, we can focus on the cost of technical-engineering building observation. Nowadays, the cost of certain works is determined by using [1].

Previously, the cost of such works was defined by the collection [2], the method of the cost calculation didn't experience significant changes up to date. At the same time, prices for some types of work, which are absent in this collection, but similar in content, were also calculated with the help of [3].

Firstly, data, on the basis of which the estimate is compiled, must be defined. It can be the amount of the work in progress, parameters of the surveyed buildings, work conditions, work changes, etc.

Secondly, we must describe the cost parameters used in the estimate. Unit and common rates, coefficients, allowances, compensations, etc. are related to these parameters.

But the order of the cash budget formation is not limited by the specific set of parameters. What is important is the method of their calculation, i.e., algorithm of the task solving with specific values usage. In addition, the parameters are divided into certain groups. For example, rates can be public or private.

Thirdly, the outcome estimate must be calculated after all of the studied parameters are figured out and taken into account. The final usage of the received parameters for determination of the final cost of works involves two moments: formation of the cost of work stages and formation of the general cost of works.

Since the parameters for estimate calculation contain two main groups: coefficients and valuations, the elements of the other blocks, i.e. data and parameter determination methods, 
will be grouped around these types of parameters. Data contain elements, that are necessary to form the parameters. Architectural volume of the building is required to form the cost parameters, that are based on the valuations. The number of such trials should be known to determine the cost formation of the physical-mechanical characteristics. Except of the influence on the cost of all trials, obtained by multiplying of the unit price and their quantity, its number also affects the valuation [4].

To determine the parameters, depending on the bench-mark data, there are special methods of their formation, which determine the arrangements of using bench-mark data to obtain the final value. Therefore, valuations can be roughly divided to public and private. They differ in the cost formation of works in section, public evaluations are presented for works, which cost is determined by multiplying of valuation by the architectural volume. Private ones form the cost of works by multiplying the valuation by the set of certain type of measurements, i.e. the carrying out of any standardized trial the n-number of times [5].

For example, public prices for the valuation of the diagnostic studies are determined by the height of the building, category of the complexity of the works and the complexity of the unit. Building height dependence doubles the valuation dependence on the total structural volume and must be inscribed, in our view, in the main input parameters for determination of the valuation along the building square. Depending on the category and complexity of construction works is quite complicated, in particular due to the fact of the difficulty to separate the factors related to the complexity of works and the factors related to the category of complexity of the building, because these factors are interchangeable and complementary. For example, emergency state of the structures increases complexity of works and it is related to the corresponding factors. But in fact, this feature determines the condition of the building and therefore represents the element of the building complexity, etc. According to these arguments, it is proposed to allocate the category of complexity of the unit, which replaces both the category of complexity of works and the category of complexity of building and includes all stated above factors [6].

Part of the cost, which is formed by the private valuations, correspondingly, depends on the method of the work performance, i.e. the kind of trials or measurements and their number.

Now the method of the price formation only remains to be specified with the help of coefficients. Coefficient accounting is performed by multiplying the costs, received with the help of valuations, and the corresponding coefficient, which reflects the method the work has been performed. For example, the cost of the survey work is multiplied by the coefficient 1.15 if the survey required the usage of ladders and equipment [7].

\section{Results}

Coefficients can also be public and private. Public coefficients are related to all types of works, private ones - only to some of them. Therefore, the presence of the valuations and coefficients determines the method the cost of the works is formed: first, the cost is created with the use of valuations, then it is corrected using coefficients.

The next stage of the studies and measurements price determination is actually a stage of obtaining the final results. Final formation of the valuation of types or stages of works and formation of the total cost of the works is performed on this stage.

For the cost per unit of total structural volume types of work it represents the obtaining of valuation production not only for public and private coefficients, applicable for this kind of works, but for the structural volume of the building as well. For the cost per measurement (trial) types of work it is necessary to take the product of the sum of the costs of separate types of trials and the used certain public or private coefficients, i.e. by their product [8]. 


\section{Discussion}

Such classification and engineering survey pattern of works allow to identify interconnections between separate elements more clearly, reflect the entire survey process more comprehensively, identify similar interdependencies and logic of the connection of separate elements, somewhat simplify the computation scheme for valuation of separate parts and the whole process in general. Selection of the bench-mark data block allows to organize the data and, may be to elaborate a simpler method of their calculation and organization $[9,10,11]$. Determination of the basic parameters of the calculation scheme both valuations and coefficients allows to focus the whole attention on them at preparation of the guidance papers, creation of the united regulatory system for calculation of the estimates or its adjustment in the direction of simplification and precision of the data processing.

\section{Conclusions}

As for the regulatory basis to determine the cost of the inspection, we offer the following:

1) in the case of public costs, it is recommended forming them depending on the height and the square of a building, but not on the height and the total structural volume, as it was performed before;

2 ) in the case of private costs, it is recommended defining the amount of valuation in regards to the number of trials, i.e. it should reduce with the increase in the number of trials or set the number of trials according to the total structural volume;

3 ) in the case of private coefficients, it is suggested implementing some additional coefficients which reflect the modern methods of work performance;

4) in the case of private and public coefficients, it is suggested clarifying the methods of their calculation for the project and survey contractors due to the work intensity, which we demonstrated before.

\section{References}

1. A. Azriyah, S. Auzair, R. Amiruddin,Procedia - Social and Behavioral Sciences, 219, 84-90, (2016)

2. S.V. Belyaeva, Vestnik MGSU, 4, 130-139, (2016).

3. G.Dosi, M.Grazzi, D.Moschella,Research Policy,44, 1795-1814, (2015).

4. E.M. Kiseleva, M.L. Nekrasova, M.A. Mayorova, M.N.Rudenko, V.S. Kankhva, International reviewof management and marketing (IRMM),6S, (2016)

5. O.Flack, G. Głód, Procedia - Social and Behavioral Sciences. 207, 608-631, (2015).

6. O.A. Gorb, I.A.Yasnolob, N.Y. Protsiuk,Organizational-economic mechanism of management, Annals of Agrarian Science, (2016)

7. H.M. Gumba, V.U. Mikhailov, V.V. Gamuletskiy. Formation of the mechanism of innovative-strategic development of construction enterprises, MSUCE, Moscow, (2011)

8. S. Pérez-Moreno, B. Rodríguez, M.Luque, Economic Modelling, 53, 398-408, (2016).

9. S.S. Uvarova, V.S. Kankhva, S.V. BelyaevaEconomic sustainability of construction companies and projects, MGSU, Moscow, (2011)

10. S.S.Uvarova, Vestnik MGSU, 8, 110-120, (2016)

11. S.S.Uvarova, Construction economics. 2, 69-77, (2014) 